\title{
EE-eICIC: Energy-Efficient Optimization of Joint User Association and ABS for eICIC in Heterogeneous Cellular Networks
}

\author{
Jie Zheng, ${ }^{1}$ Ling Gao, ${ }^{2}$ Hai Wang, ${ }^{1}$ Jinping Niu, ${ }^{1}$ Xiaoya Li, ${ }^{1}$ and Jie Ren \\ ${ }^{1}$ School of Information and Technology, Northwest University, Xian 710127, China \\ ${ }^{2}$ Xian Polytechnic University, Xian 710071, China \\ ${ }^{3}$ School of Computer Science, Shaanxi Normal University, Xian 710119, China \\ Correspondence should be addressed to Ling Gao; gl@nwu.edu.cn and Hai Wang; hwang@nwu.edu.cn
}

Received 1 May 2017; Accepted 16 July 2017; Published 7 September 2017

Academic Editor: Sergio Herrería-Alonso

Copyright (C) 2017 Jie Zheng et al. This is an open access article distributed under the Creative Commons Attribution License, which permits unrestricted use, distribution, and reproduction in any medium, provided the original work is properly cited.

\begin{abstract}
The densification and expansion of heterogeneous cellular networks (HetNets) pose new challenges on interference management and reduction of energy consumption. The $3 \mathrm{GPP}$ has proposed enhanced intercell interference coordination (eICIC) by making a macrocell silent in almost blank subframes (ABSs) to mitigate interference for low power base stations (BSs) in HetNets. However, energy efficiency $(\mathrm{EE})$ is very crucial for the deployment of a large number of low power nodes as they consume a lot of energy. In this work, we develop a novel EE-eICIC algorithm to determine the amount of ABSs and user equipment (UE) that should associate with picocells or macrocells from energy efficiency perspective. Due to the nonsmooth and mixed combinatorial features of this formulation, we focus on a suboptimal algorithm design. Using generalized fractional programming and the convex programming theory, we propose an iterative and relaxed-rounding algorithm to solve the problem. Numerical results illustrate that the proposed EE-eICIC algorithm achieves superior performance in comparison with state-of-the-art methods in terms of energy efficiency of both system and user.
\end{abstract}

\section{Introduction}

To meet the demand of prolific growth in wireless data, LongTerm Evolution-Advanced (LTE-A) has deployed low power nodes (e.g., small cells) underlying the existing high power Macro-only cellular networks [1]. Owing to the large power disparities in the downlink direction between base station (BS) types in heterogeneous cellular networks (HetNets), macrocells can cover much larger areas than small cells (e.g., femtocells or picocells) so that the macrocells can be overloaded and the small cells can be underutilized. Offloading users onto small cells is more important for tapping the potential of HetNets, called load balancing [2]. Moreover, the interference of macrocells to small cells limits the application of cell-specific dense small cells in the 5G HetNet deployment scenario [3].

To protect small cells from macrocells, the 3GPP standard has proposed the notion of enhanced intercell interference coordination (eICIC) where the macrocell should keep downlink transmission silent in certain time resources, namely, almost blank subframes (ABSs) [4]. There are two important features: user association and ABSs allocation in eICIC. Firstly, by assigning a user to a macrocell or small cell, one can ensure that small cells are not underutilized or overutilized. Secondly, a macrocell can mute all downlink transmission to its user equipment (UE) in ABS, reducing the interference from the macrocell to assist small cell transmission. Therefore, a small cell can transmit to its UE at a much higher data rate during ABS with much less interference.

The eICIC configuration problem is coupled with user association; that is, the parameters of ABS and user association rule decide the available radio resources and the users assignment between macrocell and picocell. Most existing works [5-7] have focused on different dynamic ABS configuration schemes for load balancing between macrocell 
and small cell with the dynamic variations of load. But these works almost pay more attention to improving the network throughput but neglect the energy efficiency (EE) of networks. And only ABS configuration cannot meet the need of reducing cross-tier interference significantly when the number of picocells is large [8]. The work in [9] has shown that per-tier biasing rule is not optimal for energy saving on load balancing. It reveals that the user association for energy saving is quite different from load balancing investigated for system capacity on interference management. Interference management together with reducing energy consumption should be considered jointly in HetNets [10]. Therefore, how to set the EE-eICIC parameters, that is, energy efficiency of joint UE association and ABS optimization, is left unspecified in the eICIC standard. Our goal is to promote the capacity and energy efficiency of systems while keeping the traffic load balance between macrocells and picocells. In this paper, we propose an energy-efficient joint UE association and ABSs allocation scheme for eICIC.

The main contributions of this paper are threefold. Firstly, we formulate an energy-efficient optimization problem to maximize the network energy efficiency. Secondly, we provide a formal framework for energy-efficient optimization of joint ABSs allocation and UE association in each cell by considering base station-to-base station interference maps as well as network topology. Thirdly, using the generalized fractional programming and convex optimization theory, we propose a simplified iterative and relaxed-rounding algorithm to solve this mixed-integer nonlinear programming problem.

The paper is organized as follows. Section 2 provides the related work. Section 3 introduces the HetNets model and presents the downlink interference model with ABS protocol. Section 4 constructs the EE-optimization problem for eICIC configuration. In Section 5, we design an iterative and twostep algorithm to solve the optimization problem. Numerical results are presented in Section 6. Section 7 concludes this paper.

\section{Related Work}

Energy Efficiency of UE Association. One of the major design principles to reduce energy consumption in HetNets is to allocate the load of the network to different BSs [3]. There is a high potential for energy saving if BSs can be switched off according to different traffic loads [11]. To minimize the total cost function, joint user association and BS switching on/off algorithm are designed to balance energy consumption and revenue in HetNets [12]. Reference [13] studied BS closing strategies to maximize EE or minimize total power consumption for downlink multiantenna multicarrier small cell networks supporting best effort traffic. This work in [14] derived a balance between the average traffic delivery latency and the green energy utilization with load balancing. Han and Ansari [15] considered both the traffic delivery latency and the green energy generation rate in BSs for determining user association. The technique of ABS is not considered in those works, which have only taken into account load balance but neglected the severe interference from macrocells.
Energy Efficiency of ABS Optimization. The work in [5-7] optimized the ABSs allocation for system throughput and users rate. As we know, the energy efficiency of ABS optimization mainly focused on the interference coordination between macrocells and picocells in time domain. The work [16] studied the power optimization problem in coordinated multipoint (CoMP) systems with max-min fairness. The proposed method in [17] is to allocate frequency and power resources between macrocells and picocells for power reduction and interference mitigation. To achieve energy efficiency in HetNets, multicell multiuser channel assignment should be integrated into the optimization of the cell activation and user association [9]. Also, Zhuang et al. jointly optimized energyefficient user association and spectrum allocation to reduce the energy consumption and increase the quality of service in HetNets [18].

Energy Efficiency of Joint UE Association and ABS Optimization. Most works of joint UE association and ABS allocation were proposed to maximize the system throughput and users service rate. The user association and ABS ratio issues jointly were formulated as a network-wide max-min fairness optimization problem in terms of system throughput [19] and fairness of users service rate [20]. Deb et al. [4] proposed a joint optimization framework for user association and ABSs allocation to maximize the weighted proportional fairness of users. An alternating direction method of multipliers (ADMM) algorithm was proposed to jointly optimize user association and $A B S$ ratio; the algorithms implemented in distributed manner on each Macro BS and Pico BS were coordinated to allocate average airtime for the whole network [21]. An adaptive energy-efficient ABS configuration scheme was proposed to configure the proper ABS ratio and optimal power according to the practical load [8]. However, the association of UE is predetermined with signal-to-interference plus noise ratio (SINR) in the downlink between macrocells and picocells. Zheng et al. [22] studied joint load balancing of downlink and uplink in HetNets to maximize the system capacity by a two-step relaxed-rounding method. We followed the methods of Zheng et al. (2016); however, we focus on the energy efficiency optimization of a system for downlink.

\section{System Model}

We consider a two-tier HetNets system for TDD-LTE, where the subframes for eICIC are configured dynamically. UE is user equipment (e.g., mobile device), and eNodeB indicates evolved node carrying out radio resource management, admission control, and other functionalities for BS.

User Model. For UE downlink transmission, the best candidate BSs of macrocells and picocells are determined according to the received signal strength across the entire bandwidth measured by UE. We assume that the BS has the maximum transmit power and UE associates with the BS depending on the received signal strength by all BSs including macrocells and picocells in the entire bandwidth. So, we investigate the 


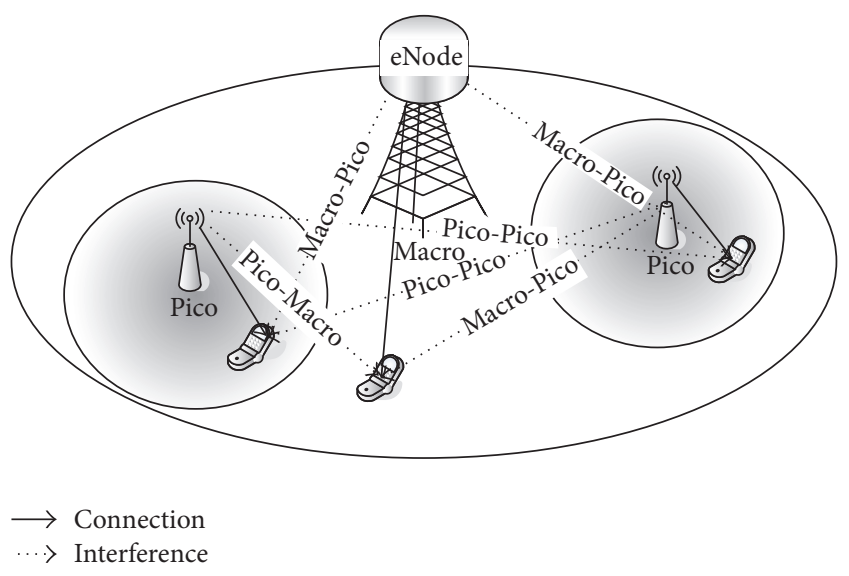

FIGURE 1: Interference model for downlink transmission in HetNets.

energy efficiency of eICIC from the perspective of lower bound.

Interference Model. For the purpose of SINR model, we distinguish downlink interference into three types of interference for Macro-to-Macro interference, Pico-to-Pico interference, and Macro-to-Pico interference as shown in Figure 1. The Macro-to-Macro interference is due to 1:1 frequency reuse in most LTE networks. For two-tier TDD-LTE, macrocells and picocells are in the same bandwidth, which we refer to as Macro-to-Pico interference. Picocells can interfere with each other in the cochannel (i.e., Pico-to-Pico interference).

eICIC ABS. The eICIC is proposed for interference coordination between macrocells and picocells in cochannel development. In order to assist the transmission of picocells in the downlink, the macrocell keeps the downlink silent for ABS and limits control signals over these subframes. In this work, we use energy-efficient ABS configuration for eICIC to improve the EE utilization of ABS and determine users association between macrocells and picocells and allocate airtime for UE from energy efficiency perspective. These are shown in Figure 2.

SINR Model. On the basis of the interference model, we derive the SINR model. Then, the average data rate of UE can be computed by Shannon's capacity formulation. A list of parameters used in the SINR model are shown in the Notation. The interfering maps between BSs can be determined by the physical distance or received signal strength from one BS to another BS whether or not it is larger than threshold setting.

Since UE only associates with macrocells or picocells but not both simultaneously in the downlink, UE is classified into two types: Pico-associated and Macro-associated. For Pico-associated UE, the UE can transmit for both ABS and non-ABS. The interference to the Pico-associated UE is from picocells but not from macrocells since the macrocell keeps silent for the downlink in ABS. For Macro-associated UE, the $\mathrm{UE}$ can only transmit for non-ABS. The interference to the Macro-associated UE is from picocells and macrocells. So, the SINR of UE can be calculated in the following.
For Pico-associated UE, the downlink SINR of UE $u$ can be modeled as

$$
\begin{aligned}
& \operatorname{SINR}_{\text {pico }}(u) \\
& = \begin{cases}\frac{P_{R x}(u)}{P_{\text {pico }}(u)+N_{0}} & \text { for ABS subframes } \\
\frac{P_{R x}(u)}{P_{\text {pico }}(u)+P_{\text {macro }}(u)+N_{0}} & \text { for non-ABS subframes. }\end{cases}
\end{aligned}
$$

For Macro-associated UE, the downlink SINR of UE $u$ can be obtained with

$$
\operatorname{SINR}_{\text {macro }}(u)=\frac{P_{R x}(u)}{P_{\text {pico }}(u)+P_{\text {macro }}(u)+N_{0}}
$$

for non-ABS subframes.

Then, we can get the average data rate for UE $u$ from SINR model, which are denoted in the Notation.

\section{Optimization Problem Formulation}

We will find an algorithm to maximize the energy efficiency of the system for eICIC in HetNets. Therefore, we formulate this problem as three optimization choices:

(1) UE EE association for downlink; that is, UE associates with the best BS (macrocell or picocell) in the downlink from EE perspective.

(2) The optimal EE-ABS number, which be used for interfering picocells by each macrocell so that picocells can be protected from macrocell interference.

(3) The time-average airtime of UE; that is, the time scale of subframes per ABS period across the entire bandwidth for user referring to the load of $\mathrm{BS}$ determines the rate and energy consumption of each user per ABS period.

The optimization variables and parameters are summarized in the Notation.

4.1. Problem Formulation. Naturally, to maximize the EE of the network, we jointly optimize these variables $\psi=\left\{R_{u}, P_{u}\right.$, $\left.x_{u}, y_{u, A}, y_{u, n A}, A_{p}, N_{m}\right\}$ to obtain the EE-eICIC algorithm. The optimization problem (OP1) is modeled as follows:

$$
\begin{aligned}
& \max _{\psi} \frac{\sum_{u} R_{u}}{\sum_{u} P_{u}} \\
& R_{u} \leq r_{u}^{\mathrm{macro}} \cdot x_{u}+r_{u, A}^{\mathrm{pico}} \cdot y_{u, A}+r_{u, n A}^{\mathrm{pico}} \cdot y_{u, n A} \\
& P_{u} \leq p_{u}^{\mathrm{macro}} \cdot x_{u}+\left(p_{u}^{\mathrm{pico}}+P_{\mathrm{ref}}^{\mathrm{macro}}\right) \cdot y_{u, A}+p_{u}^{\mathrm{pico}} \\
& \quad \cdot y_{u, n A} \\
& x_{u} \cdot\left(y_{u, A}+y_{u, n A}\right)=0 \\
& A_{p}+N_{m} \leq N_{\mathrm{sf}}, \quad \forall p, m \in I_{\mathrm{BS}} \\
& \sum_{u \in U_{m}} x_{u} \leq N_{m}, \quad \forall m \in M \\
& \sum_{u \in U_{p}} y_{u, A} \leq A_{p}, \quad \forall p \in P
\end{aligned}
$$




\begin{tabular}{|c|c|c|c|c|c|c|c|c|c|c|}
\hline \multirow[b]{2}{*}{ Macro } & $\begin{array}{c}\text { Subframe } \\
(1 \mathrm{~ms})\end{array}$ & \multicolumn{3}{|c|}{$\stackrel{\mathrm{ABS}}{\longleftarrow}$} & \multicolumn{3}{|c|}{ ABS } & \multicolumn{3}{|c|}{ ABS } \\
\hline & $\begin{array}{l}\text { Macro } \\
\text { Tx }\end{array}$ & $\begin{array}{c}\text { Macro } \\
\text { Tx }\end{array}$ & $\begin{array}{l}\text { Macro } \\
\text { silent }\end{array}$ & $\begin{array}{c}\text { Macro } \\
\text { Tx }\end{array}$ & $\begin{array}{c}\text { Macro } \\
\text { Tx }\end{array}$ & $\begin{array}{l}\text { Macro } \\
\text { silent }\end{array}$ & $\begin{array}{l}\text { Macro } \\
\text { Tx }\end{array}$ & $\begin{array}{c}\text { Macro } \\
\text { Tx }\end{array}$ & $\begin{array}{c}\text { Macro } \\
\text { silent }\end{array}$ & $\begin{array}{l}\text { Macro } \\
\text { Tx }\end{array}$ \\
\hline Pico & $\begin{array}{c}\text { Not only } \\
\text { Pico Tx }\end{array}$ & $\begin{array}{c}\text { Not only } \\
\text { Pico Tx }\end{array}$ & \begin{tabular}{|} 
Only \\
Pico Tx
\end{tabular} & $\mid \begin{array}{c}\text { Not only } \\
\text { Pico Tx }\end{array}$ & $\mid \begin{array}{c}\text { Not only } \\
\text { Pico Tx }\end{array}$ & \begin{tabular}{|c|} 
Only \\
Pico Tx
\end{tabular} & $\begin{array}{l}\text { Not only } \\
\text { Pico Tx }\end{array}$ & $\begin{array}{l}\text { Not only } \\
\text { Pico Tx }\end{array}$ & $\begin{array}{c}\text { Only } \\
\text { Pico Tx }\end{array}$ & $\begin{array}{c}\text { Not only } \\
\text { Pico Tx }\end{array}$ \\
\hline & 1 & 2 & 3 & 4 & 5 & 6 & 7 & 8 & 9 & 10 \\
\hline
\end{tabular}

Figure 2: ABS subframe.

$$
\begin{aligned}
& \sum_{u \in U_{p}} y_{u, A}+y_{u, n A} \leq N_{\mathrm{sf}}, \quad \forall p \in P \\
& x_{u} \geq 0, \\
& y_{u, A} \geq 0, \\
& y_{u, n A} \geq 0 \\
& A_{p}, N_{m} \leq N^{+}, \quad \forall p, m \in I_{\mathrm{BS}},
\end{aligned}
$$

where $\mathrm{N}^{+}$is the set of nonnegative integers.

Constraint (4) states that the average rate for a user cannot be more than what is available based on the airtime from the associated macrocell or picocell. Formulation (5) states that the average power consumption for a user cannot be more than the available airtime from the associated macrocell or picocell. The association constraint (6) denotes that UE only associates with either macrocell or picocell, but not both. Constraint (7) ensures that the ABS subframes used by picocells are provided by macrocells in $I_{\mathrm{BS}}$ which keep silent to decrease interference to picocells, where $I_{\mathrm{BS}}$, BS $\in\{$ macrocell, picocell $\}$, denotes the set of all interfering picocells and all interfering macrocells (i.e., BSs interfere with each other). Constraint (8) states that time scale of subframes allocated to UE from a macrocell can be less than the total available ABS subframes $N_{m}$. Constraint (9) states that the time scale of ABS subframe allocated to the UE from a picocell is less than the total available ABS subframes $A_{p}$. Constraint (10) states that the time scale of subframe allocated to UE from a picocell is less than the total ABS period $N_{\mathrm{sf}}$. This also means that the total average airtime allocated to UE from a macrocell or a picocell is less than the total usable subframes.

Remark 1. Constraint (6) ensures that UE associates with single macrocell or picocell; OP1 is a binary integer programming problem. Moreover, since (11) involves continuous variables $R_{u}, P_{u}, x_{u}, y_{u, A}, y_{u, n A}$ and (12) contains nonnegative integer variables $A_{p}, N_{m}$, the OP1 is a mixed binary integer programming problem. Finding the optimal solution to OP1 is generally NP-hard [23]. In this paper, we solve it in a different way.
4.2. Problem Transformation. Due to constraints (6) and (12), the OP1 is a mixed binary integer programming problem. However, even if we remove constraint (6) and relax (12) to $R^{+}$, the OP1 is still nonconvex owing to the nonconvexity of the objective function (3). We first exploit the structure of (3) to reformulate with generalized fractional programming [24].

Without loss of generality, we assume that $R_{u}>0$ and $P_{u}>0$. For notational simplicity, we denote the feasible region of (4)-(12) in OP1 by $\psi$. So,

$$
\eta_{\mathrm{EE}}^{\mathrm{opt}}=\max _{\psi^{\mathrm{opt}}} \frac{R_{u}^{\mathrm{opt}}}{P_{u}^{\mathrm{opt}}}=\frac{R_{u}^{\mathrm{opt}}}{P_{u}^{\mathrm{opt}}},
$$

where $\psi^{\mathrm{opt}}=\left\{R_{u}^{\mathrm{opt}}, P_{u}^{\mathrm{opt}}, x_{u}^{\mathrm{opt}}, y_{u, A}^{\mathrm{opt}}, y_{u, n A}^{\mathrm{opt}}, A_{p}^{\mathrm{opt}}, N_{m}^{\mathrm{opt}}\right\}$ and $\eta_{\mathrm{EE}}^{\mathrm{opt}}$ are the optimal solution of OP1, respectively.

To solve OP1, we give the following proposition. The proof uses a standard result in the generalized fractional programming theory [25].

Proposition 2. The optimal solution $\psi^{o p t}$ is achieved if and only if

$$
\max _{\psi^{o p t}}\left(R_{u}-\eta_{E E}^{o p t} P_{u}\right)=R_{u}^{o p t}-\eta_{E E}^{o p t} P_{u}^{o p t}=0
$$

Proposition 2 indicates that we can solve OP1 via its equivalent problem (14). However, $\eta_{\mathrm{EE}}^{\mathrm{opt}}$ is generally unknown in advance. From [25], the optimal solution of OP1 can be obtained by solving (14) with $\eta_{\mathrm{EE}}^{\mathrm{opt}}$ replaced by an update parameter $\eta$. The details of the procedure are shown in Algorithm 1. Note that the optimization problem (OP2) that needs to be solved by Algorithm 1 for a given $\eta$ (e.g., $\eta_{k}$ at iteration $k$ ) is

$$
\begin{array}{cc}
\max _{\psi} & \left(R_{u}-\eta P_{u}\right) \\
\text { s.t. } & (4)-(12) .
\end{array}
$$

However, solving (15) is still hard for a given $\eta$. Even in a single Pico and a single interfering Macro, the OP2 problem is also NP-hard [4]. Thus, fast-convergent suboptimal algorithms are preferred in practice. 


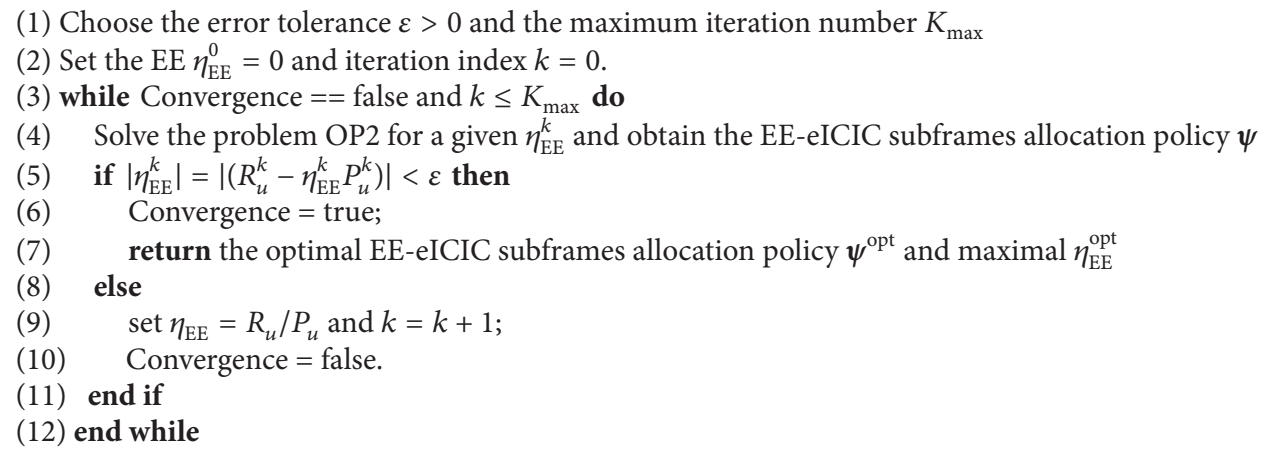

Algorithm 1: Iterative algorithm for EE-eICIC.

\section{Algorithm for Relaxed-Rounding Nonlinear Program}

In this section, we followed the methods of Zheng et al. [22] to solve the OP2 problem by a two-step algorithm in polynomial time. Firstly, the integer variables can be relaxed into the nonnegative real numbers. Then, we can solve the relaxed problem easily, which is convex. Further, we obtain a feasible solution to the OP3 by rounding the output of the relaxed problem. The descriptions are as follows in detail.

(1) Relaxing. Solve the relaxed problem OP3 from OP2. The OP3 is obtained by ignoring constraint (6) and relaxing the integrality constraint (12) on $N_{m}$ and $A_{p}$. After relaxing constraint (12), $N_{m}$ and $A_{p}$ can be taken as nonnegative real numbers. Note that removing constraint (6) has no effect on the problem, which means that UE can receive radio resources from macrocells and picocells simultaneously in the downlink. The OP3 with optimization variables $\widetilde{\psi}=$ $\left\{\widetilde{R}_{u}, \widetilde{P}_{u}, \widetilde{x}_{u}, \tilde{y}_{u, A}, \widetilde{y}_{u, n A}, \widetilde{A}_{p}, \widetilde{N}_{m}\right\}$ can be modeled as

$$
\begin{array}{cc}
\max _{\psi} & \widetilde{R}_{u}-\tilde{\eta} \widetilde{P}_{u} \\
\text { s.t. } & (4)-(5), \\
& (7)-(11)
\end{array}
$$

$$
A_{p}, N_{m} \in R^{+}, \forall p, m \in I_{\mathrm{BS}},
$$

where $R^{+}$is the set of nonnegative real numbers.

(2) Rounding. In the second step, we appropriately round the output of the relaxed algorithm to yield a feasible solution to OP3.

5.1. Solution for the Relaxed Problem OP3. The relaxed OP3 is convex programming, so we can solve the convex problem OP3 in many ways like in [26]. In this paper, we introduce the CVX tools [27] to solve the OP3, which is defined as Algorithm 2. So, Algorithm 2 can be considered as the CVX. It is easy to solve the convex OP3 with the CVX formulation (16) directly. So, we simply describe the algorithm as shown in Algorithm 2.
(1) Set the OP3 with CVX formulation as the (16).

(2) Solve the problem OP3 with CVX tool directly.

Algorithm 2: Solution for the relaxed problem OP3.

5.2. Integer-Rounding the Output of Algorithm 2. Different from the relaxed problem (OP3), the downlink of one user only associates with a macrocell or picocell but not both. This means that either macrocells or picocells can transmit the data with a user in the downlink direction for per-ABS period. To obtain a feasible solution of $N_{m}$ and $A_{p}$ for the OP2, we adopt the rounding method:

$$
\text { Round }(x)= \begin{cases}\text { floor }(x) & x<\frac{N_{\mathrm{sf}}}{2} \\ \operatorname{ceil}(x) & x \geq \frac{N_{\mathrm{sf}}}{2},\end{cases}
$$

where floor is rounding down and ceil is rounding up. Then, the feasible solution can be determined approximatively by rounding the output of Algorithm 2. The rounding and UE association scheme is shown in Algorithm 3.

Algorithm 3 includes three parts. Firstly, we obtain $N_{m}$ and $A_{p}$ integer values by the defined rounding function. Secondly, the UE EE association has been determined by the comparison of the EE obtained by UE rate and energy consumption. For equation (d) in Algorithm 3, $X_{m}$ denotes the non-ABS utilization for macrocells, $Y_{p, n A}$ and $Y_{p, A}$ denote the non-ABS and ABS utilization for picocells, and $U_{m}^{*}$ is the set of users to associate with macrocells, and so is $U_{p}^{*}$ for picocells. Thirdly, each user available average airtime for downlink is computed by filling up the available subframes so that we can obtain the rate and energy consumption of the user. The EE of the system is obtained as $\eta_{\mathrm{EE}}^{*}=\sum_{u} R_{u} / \sum_{u} P_{u}$.

5.3. Performance Analysis of the Proposed Algorithm. We illustrate the performance of the proposed algorithm for the worst case through theoretical analysis. The optimal solution of the original problem is achieved with Algorithm 1, and then 
(1) EE-ABS Rounding. To take $N_{m}^{*}$ and $A_{p}^{*}$ integer values.

where $\widetilde{N}_{m}$ and $\widetilde{A}_{p}$ is output of Algorithm 2 .

$$
N_{m}^{*}=\operatorname{Round}\left(\widetilde{N}_{m}\right), \quad A_{p}^{*}=\operatorname{Round}\left(\widetilde{A}_{p}\right),
$$

(2) UE EE-Association for Downlink

$$
\begin{aligned}
& R_{u}^{\text {macro }}=r_{u}^{\text {macro }} \cdot \tilde{x}_{u}, \quad P_{u}^{\text {macro }}=p_{u}^{\text {macro }} \cdot \tilde{x}_{u} \quad(\mathrm{~b}) \\
& R_{u}^{\text {pico }}=r_{u, A}^{\text {pico }} \cdot \tilde{y}_{u, A}+r_{u, n A}^{\text {pico }} \cdot \tilde{y}_{u, n A}, \quad P_{u}^{\text {pico }}=\left(p_{u}^{\text {pico }}+P_{\text {ref }}^{\text {macro }}\right) \cdot \tilde{y}_{u, A}+p_{u}^{\text {pico }} \cdot \tilde{y}_{u, n A},
\end{aligned}
$$

where $\tilde{x}_{u}, \tilde{y}_{u, A}, \tilde{y}_{u, n A}$ is output of Algorithm 2.

Computing $\eta_{u}^{\text {pico }}=R_{u}^{\text {pico }} / P_{u}^{\text {pico }}, \eta_{u}^{\text {macro }}=R_{u}^{\text {macro }} / P_{u}^{\text {macro }}$. If $\eta_{u}^{\text {macro }}>\eta_{u}^{\text {pico }}$, UE associates with macrocell, or with picocell.

(3) Energy Efficiency Computation

Firstly, calculate non-ABS and ABS utilization of downlink.

$$
\begin{aligned}
& X_{m}=\sum_{u \in U_{m}^{*}} \tilde{x}_{u} \quad(\mathrm{~d}) \\
& Y_{p, A}=\sum_{u \in U_{p}^{*}} \tilde{y}_{u, A}, Y_{p, n A}=\sum_{u \in U_{p}^{*}} \tilde{y}_{u, n A},
\end{aligned}
$$

Secondly, for each user, calculate the time scale of subframe for downlink

$$
\begin{aligned}
& \widehat{x}_{u}=\frac{\tilde{x}_{u} \cdot N_{m}^{*}}{X_{m}} \quad(\mathrm{f}) \\
& \hat{y}_{u, A}=\frac{\widetilde{y}_{u, A} \cdot A_{p}^{*}}{Y_{p, A}} \quad(\mathrm{~g}) \\
& \widehat{y}_{u, n A}=\frac{\tilde{y}_{u, n A} \cdot\left(N_{\mathrm{sf}}-A_{p}^{*}\right)}{Y_{p, n A}}
\end{aligned}
$$

Finally, the rate and power consumption of user can be computed.

For $u \in U_{m}^{*}, R_{u}^{*}=r_{u}^{\text {macro }} \cdot \widehat{x}_{u}, P_{u}^{*}=p_{u}^{\text {macro }} \cdot \widehat{x}_{u}$.

For $u \in U_{p}^{*}, R_{u}{ }^{*}=r_{u, A}^{\text {pico }} \cdot \widehat{y}_{u, A}+r_{u, n A}^{\text {pico }} \cdot \widehat{y}_{u, n A}, P_{u}^{*}=\left(p_{u}^{\text {pico }}+P_{\text {ref }}^{\text {macro }}\right) \cdot \widehat{y}_{u, A}+p_{u}^{\text {pico }} \cdot \widehat{y}_{u, n A}$.

So the EE of user: $\eta_{u}^{*}=R_{u}^{*} / P_{u}^{*}$.

Algorithm 3: The input of rounding and association algorithm is the output of Algorithm 2.

Algorithm 2 can solve the convex problem with the CVX tool. So, the performance of the proposed algorithm is dependent on the output of Algorithm 3, which is obtained from integer-rounding the output of Algorithm 2. This is shown in the following proposition. Let $\eta^{*}$ be the EE computed by Algorithm 3 and let $\eta^{\text {opt }}$ be the optimal EE.

Proposition 3. Algorithm 3 obtains a feasible solution for the problem OP3. Furthermore, for any given $\sigma>0$, no gap exists for the CVX such that if one uses Algorithm 3 to the output of Algorithm 2 with integer-rounding operation, then

$$
2(1+\sigma) \eta^{*} \geq \eta^{o p t}
$$

Proof. See the Appendix.

5.4. Implementation Framework. The proposed EE-eICIC configuration scheme is implemented in a centralized manner. The implemented framework of the proposed EE-eICIC configuration is shown in Figure 3; dedicated Access-Stratum (AS) bidirectional connection and downlink (DL) data transmission are maintained for macrocells and picocells in radio access networks (RAN). Downlink data and Non-AccessStratum (NAS) signaling can be directly routed from picocells and macrocells to Mobility Management Entity (MME). MME can be responsible for establishing and computing the results of the proposed algorithm. Then, macro-eNodeB (eNB) carries out coordination between disparate BSs for ABSs allocation. Each BS (i.e., macrocell and picocell) divides radio resource among users associated with the different BS.
The eNodeB in RAN requires the overhead to be handled via $\mathrm{X} 2$ interface. Since the signaling and data of macrocells and picocells are directly routed to the centralized processing unit (i.e., MME), the proposed algorithm can be performed in a more efficient way.

Similar to the related work $[4,21]$, we can adjust the EEeICIC parameters for a long time period. Therefore, the ABSs allocation and the UE scheduling process in our problem are at a slow time, and the algorithm can be executed online and the results are used as a guidance for real-time scheduling.

\section{Numerical Results}

In this section, we evaluated the proposed algorithm with some simulation results. The parameters in our model are summarized in Table 1 . For the purpose of this evaluation, we selected an area of $300 \mathrm{~m} \times 300 \mathrm{~m}$ for a two-tier network with macrocells and picocells. The locations of Macro BS is fixed at the center, and the density of Macro BS is $1 / 300^{2}$. The densities of picocells and users are specified according to the different scenarios. The Pico BSs and users are produced based on a uniform distribution, with a $10 \mathrm{~m}$ constraint on minimum distance between UE and BS. Note that each point in the following simulation results is obtained by averaging 100 independent runs.

6.1. Convergence of Algorithm. In Figure 4, we plot the convergence evolution of the outer loop of EE-eICIC algorithm under the system of one macrocell, two picocells, and thirty 


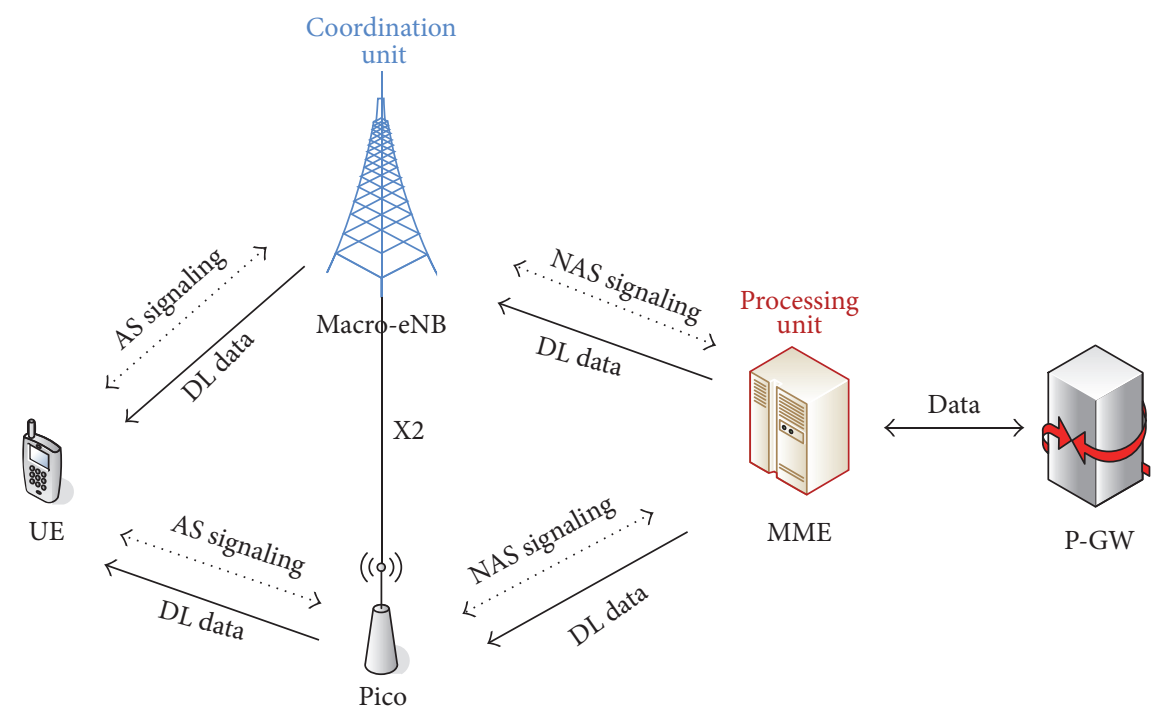

FIGURE 3: The implemented framework of the proposed EE-eICIC configuration.

TABLE 1: Simulation parameters.

\begin{tabular}{lc}
\hline Notation description & Value \\
\hline Tx power of Macros & $36 \mathrm{dBm}(4 \mathrm{~W})$ \\
Tx power of Picos & $30 \mathrm{dBm}(1 \mathrm{~W})$ \\
Broadcast signals power from Macro & $23 \mathrm{dBm}(200 \mathrm{~mW})$ \\
during ABS subframes & $-174 \mathrm{dBm} / \mathrm{Hz}$ \\
Thermal noise power & 3.25 \\
Path-loss exponent & 40 \\
$N_{\text {sf }}$ frame & $10 \mathrm{MHz}$ \\
Bandwidth &
\end{tabular}

users. It is observed that it converges typically in ten steps. We solve the relaxed fractional programming for a given $\eta_{\mathrm{EE}}^{k}$ by using CVX which can be implemented in a centralized manner, that is, Algorithm 2. Thus, Figure 4 shows the overall convergence rate of EE-eICIC algorithm, which is cost-efficient in the computational complexity.

6.2. Performance Improvement from EE-eICIC. For comparison, the three methods we use are as follows.

(1) Max sum rate with eICIC (MaxSUMRate) [19]: the user in LTE associated with the maximum sum rate for eICIC can lead to load balancing between macrocells and picocells. With load balancing of eICIC, we can optimize ABS and user association so that the offloaded users can receive much higher SINR from picocells.

(2) Max log rate with eICIC (MaxSUMLogRate) [4]: the function $\ln \left(R_{u}\right)$ is to maintain proportional fairness for UE. Max $\ln \left(R_{u}\right)$ with ABS is the solution for load-aware association scheme to maximize the system throughput with UE rate fairness. Thus, we compare the ABS allocation of load-aware association with our proposed method.

(3) Proposed method Max EE with eICIC (MaxEE): the proposed method with EE-eICIC is presented to obtain

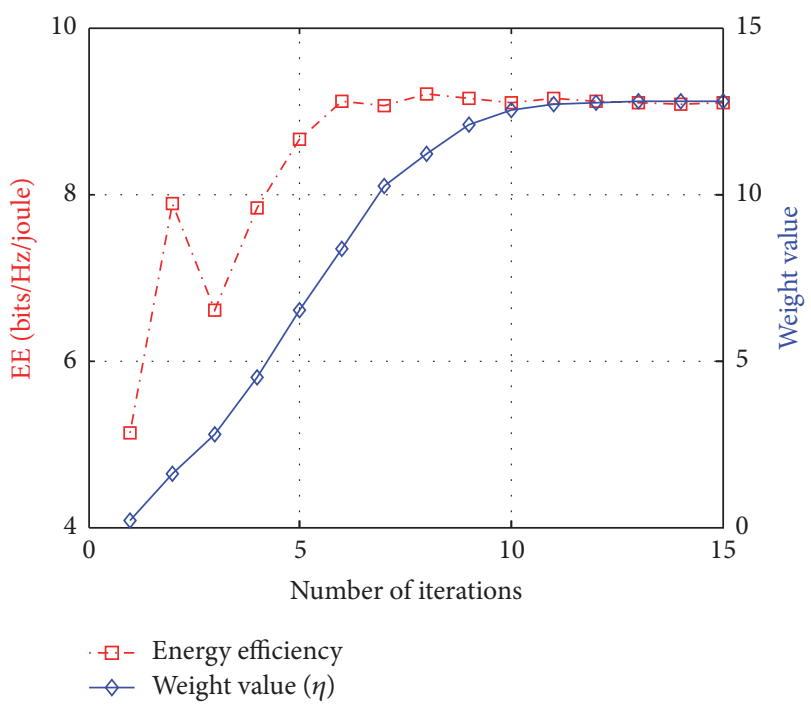

FIGURE 4: Convergence of the outer loop of EE-eICIC algorithm.

energy-efficient ABSs allocation and UE association between picocells and macrocells.

Thus, we compare the ABSs allocation of load-aware association with our proposed algorithm.

Figure 5 shows the effect of the number of users on energy efficiency of the system, corresponding to the number of macrocells of 1 and the number of picocells of 2. In Figure 3, it can be seen that MaxEE achieves significant energy efficiency gain over MaxSUMLogRate and MaxSUMRate. The proposed scheme MaxEE can improve the network energy efficiency performance by an average of $21.4 \%$ and $43.6 \%$, compared to MaxSUMRate and MaxSUMLogRate, respectively. In particular, the proposed scheme MaxEE can obtain more gain than MaxSUMLogRate and MaxSUMRate with the increasing number of users. We can see that the eICIC needs to be designed from an energy efficiency perspective. 


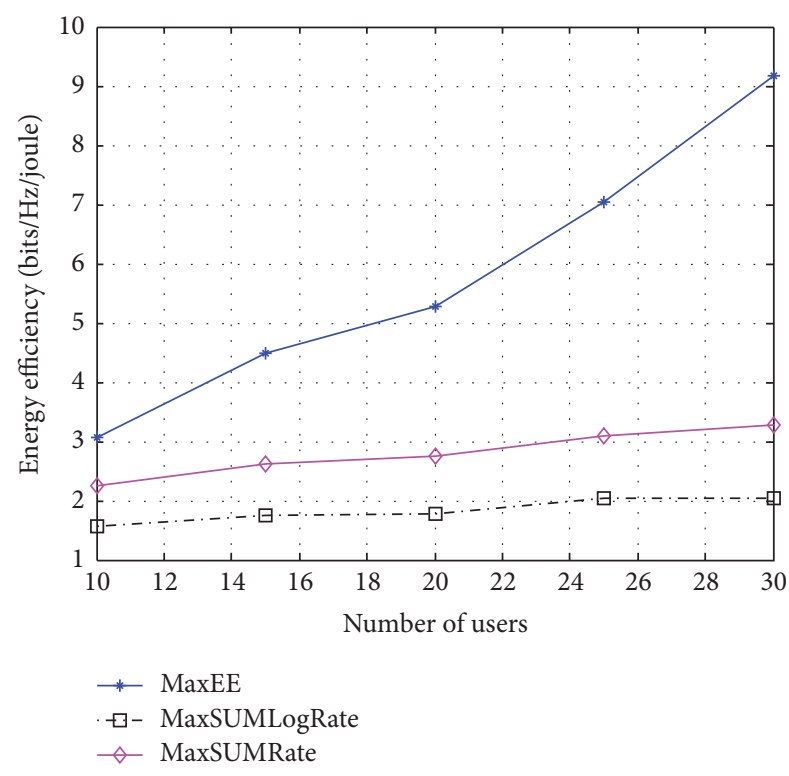

FIGURE 5: Energy efficiency versus number of users.

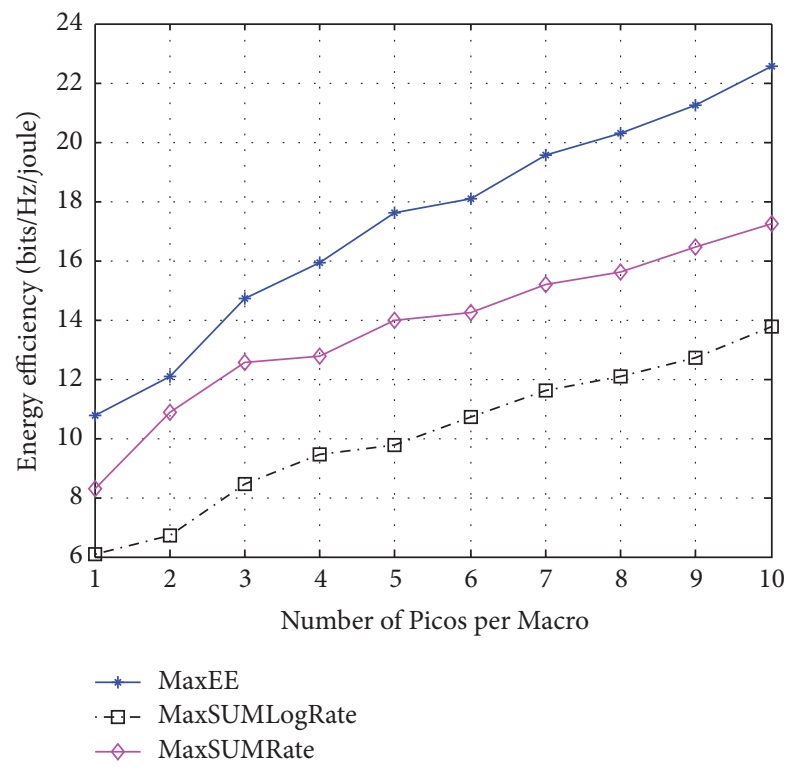

FIGURE 6: Energy efficiency versus number of picocells.

In Figure 6, we illustrate the effect of the number of picocells on energy efficiency in a thirty-user system of one Macro; that is to say, a thirty-user uniform distribution for $300 \mathrm{~m} \times 300 \mathrm{~m}$ is a nominal UE density of around 330 active UE per $\mathrm{km}^{2}$. It can be seen that the energy efficiency achieved by the proposed scheme MaxEE obtains the best performance, which has the EE gain of about $23.44 \%$ and 64.71\% over the MaxSUMRate and MaxSUMLogRate on average. Moreover, the EE gain increases with the number of picocells, which means that small cells are energy-efficient for HetNets. For ultradense HetNets in the future, it will be more energy-efficient. This is because the distance between BSs and UE becomes more close.

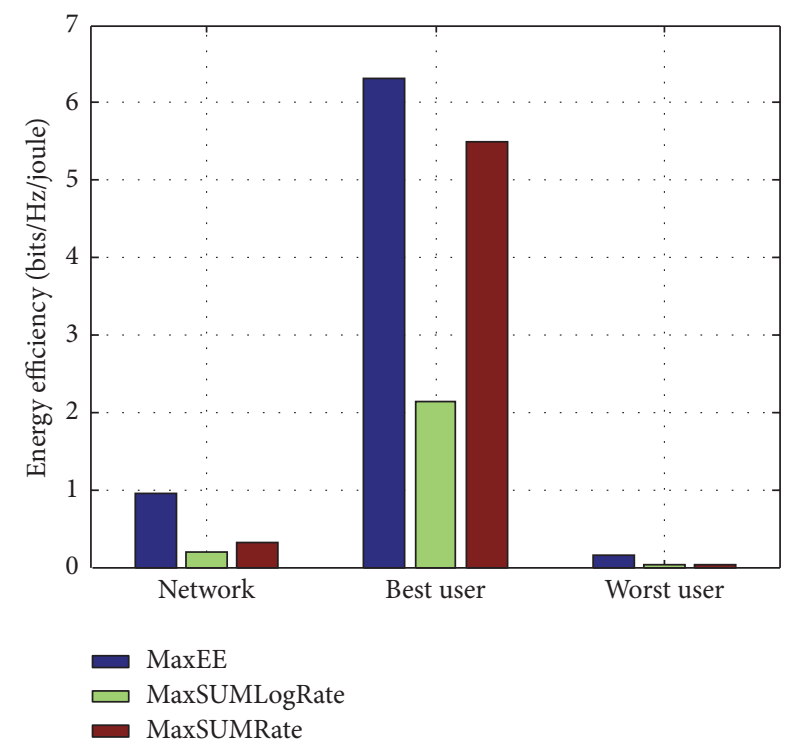

FIGURE 7: Energy efficiency of the network, the best UE, and the worst UE.

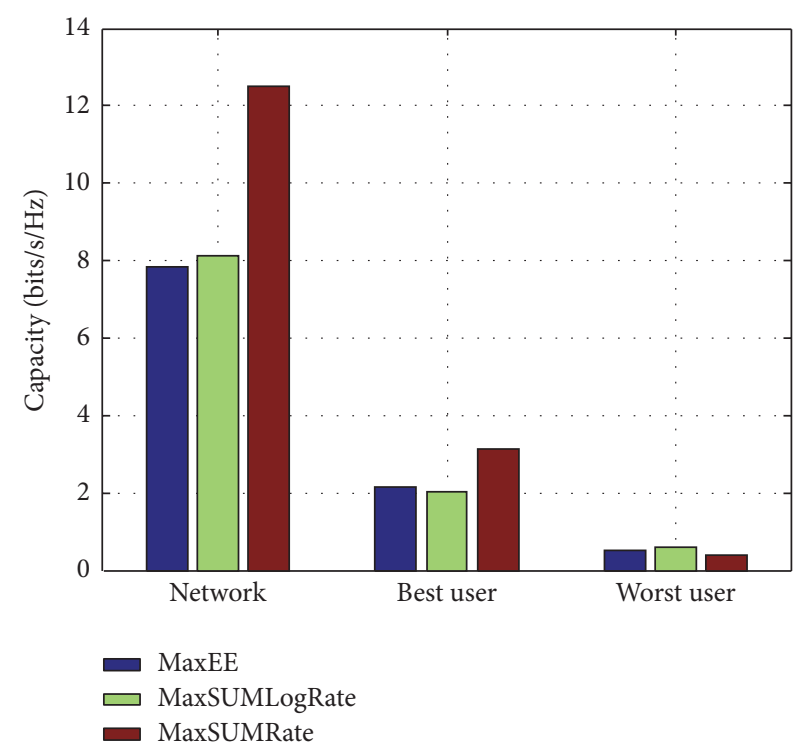

FIGURE 8: Capacity of the network, the best UE, and the worst UE.

In Figure 7, we further provide the performance comparisons among these schemes from three aspects: the energy efficiency of network, the best UE, and the worst-case UE corresponding to one macrocell, two picocells, and thirty users. We observe that there is a considerable gain in energy efficiency between the best and the worst UE in the MaxEE.

In addition, we plot Figure 8 to show the rate performance of MaxEE in comparison to the other two algorithms in the same scenarios of Figure 7. We can see that the MaxEE achieves the lowest rate of the network, but the rates of the best UE and the worst-case UE are not the lowest. This is due to the fact that MaxSUMRate is to maximize the throughput of the system, and the MaxSUMLogRate is to 


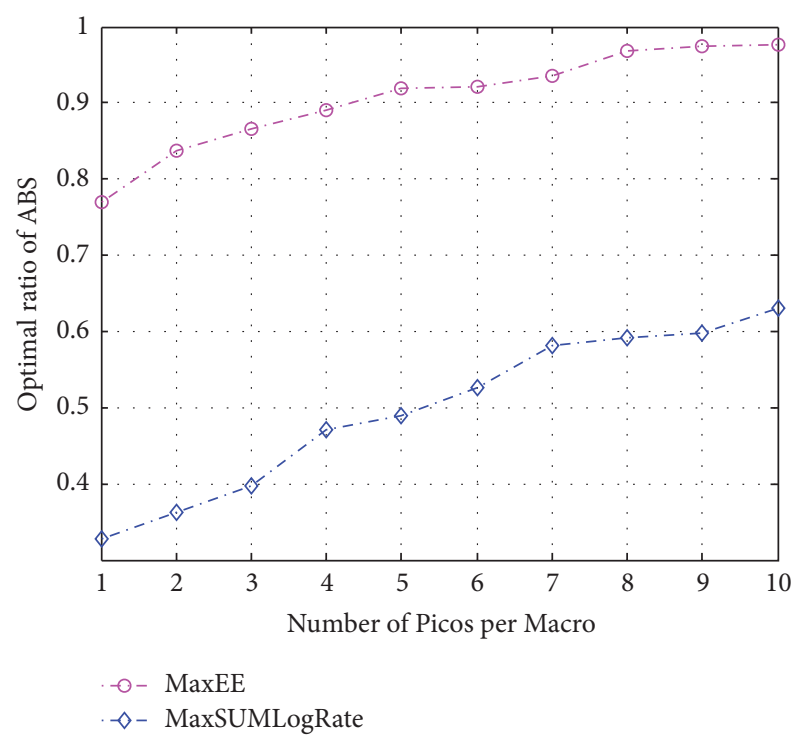

FIgURE 9: Optimal ratio of ABS versus the number of picocells.

strike a very good balance between system throughput and UE rate fairness. It is observed from Figures 7 and 8 that the energy efficiency improves at the cost of the total sum rates for MaxEE as compared with the MaxSUMRate and MaxSUMLogRate schemes.

Figure 9 gives the impact of different densities of picocells on the optimal ratio of ABS in a thirty-user system for the MaxSUMLogRate and the proposed scheme MaxEE in HetNet. In Figure 9, it can be shown that the optimal ratios of MaxSUMLogRate and MaxEE augment when picocells become denser, but the optimal ratio of the proposed scheme MaxEE is larger than the MaxSUMLogRate. This is due to the fact that the picocell is more energy-efficient. The MaxEE increases the transmission for picocells and the ABS will become larger. When the density of picocells increases, more users associate with picocells for MaxEE. Hence, EE-eICIC makes full use of the benefits of small cells, and the EE-eICIC is more energy-efficient for picocells when the small cells become denser.

\section{Conclusion}

In this paper, we have proposed a novel energy-efficient optimization framework for eICIC in HetNets to solve this mixed-integer association and ABS allocation in a two-step relaxed-rounding algorithm to further reduce the computational cost. Our numerical results verified the convergence performance of the proposed algorithm. The simulation results show that joint UE EE association and EE-ABS allocation can achieve a significant gain on energy efficiency of the system and users, but this is at the cost of the total throughput of the network. In addition, the fairness and distributed algorithm is more important for ultradense HetNets. Future work could include EE-eICIC that incorporates dynamic traffic and high user mobility with the consideration of fairness and the distributed implementation.

\section{Appendix}

\section{Proof of Proposition 3}

We provide a simple proof from basic algebraic manipulations in [4]. We define the rounding function $\operatorname{Round}(x)$ in (17), which satisfies the following.

Lemma A.1. Since $x_{1}+x_{2} \leq N_{\mathrm{sf}}$, then $\operatorname{Round}\left(x_{1}\right)+$ $\operatorname{Round}\left(x_{2}\right) \leq N_{\mathrm{sf}}$. And one has $x\left(1-2 / N_{\mathrm{sf}}\right) \geq \operatorname{Round}(x) \leq$ $x\left(1+2 / N_{\mathrm{sf}}\right)$.

We use the iterative algorithm for EE-eICIC and CVX tool so that the constraint violations are at most $\sigma^{\prime}$ (this is possible by Algorithms 1 and 2). Consider a Pico; it is supposed that $A_{p} \geq 1$. Then, for any UE associated with Pico, we have

$$
\begin{aligned}
y_{u, A}^{*} & =\frac{\tilde{y}_{u, A} \cdot A_{p}^{*}}{Y_{p, A}} \geq \frac{\tilde{y}_{u, A} \cdot \widetilde{A}_{p}}{Y_{p, A}}\left(1-\frac{2}{N_{\mathrm{sf}}}\right) \\
& \geq \frac{\tilde{y}_{u, A} \cdot \widetilde{A}_{p}}{A_{p}+\sigma^{\prime}}\left(1-\frac{2}{N_{\mathrm{sf}}}\right) \geq \frac{\tilde{y}_{u, A}}{1+\sigma^{\prime}}\left(1-\frac{2}{N_{\mathrm{sf}}}\right),
\end{aligned}
$$

where we have made use of Lemma A.1.

By substituting $\sigma^{\prime}$ appropriately with a function of $\sigma$, we can obtain $y_{u, A}^{*} \geq \widetilde{y}_{u, A} /(1+\sigma)$. It can be also easily proved that the same inequality is held for the case $A_{p}<1$. Moreover, since the UE association process ensures that each UE gets associated with a picocell only if the EE of UE obtained from the picocell is larger,

$$
2(1+\sigma) \eta_{u}^{*} \geq \tilde{\eta}_{u} .
$$

For UE associated with macrocells, we can have similar results. This follows from the fact that optimal proportion solution of EE-ABS-RELAXED is better than the optimal solution for this problem.

\section{Notation}

List of Parameters

$P_{R x}(u): \quad$ The received power of UE $u$ in the downlink direction

$P_{\text {pico }}(u)$ : The received downlink interference from all other picocells

$P_{\text {macro }}(u)$ : The received downlink interference from all other macrocells

$r_{u}^{\text {macro }}$ : Downlink data rate of UE $u$ from Macro during the non-ABS subframes

$r_{u, A}^{\text {pico }}: \quad$ Downlink data rate of UE $u$ from Pico during the ABS subframes

$r_{u, n A}^{\text {pico }}: \quad$ Downlink data rate of UE $u$ from Pico during the non-ABS subframes.

Notation of Parameters and Optimization Variables

$U$ : Set of UE

$m$ : Acronym of Macro 
p: $\quad$ Acronym of Pico

$m_{u}$ : The best candidate Macro of UE $u$

$p_{u}$ : The best candidate Pico of UE $u$

$N_{\text {sf }}$ : Period of ABS, which denotes the number of total subframes

$N_{m}$ : ABS subframes used by Macro $m$, which denotes the number of subframes for which Macro $m$ can transmit in the downlink direction

$A_{p}: \quad$ ABS subframes used by Pico $p$, which denotes the number of subframes for which Pico $p$ can transmit but Macro $m$ keeps silent in the downlink direction

$x_{u}$ : $\quad$ Time scale in non-ABS subframes UE $u$ got from $m_{u}$

$y_{u, A}: \quad$ Time scale in ABS subframes UE $u$ got from $p_{u}$

$y_{u, n A}$ : Time scale in non-ABS subframes UE $u$ got from $p_{u}$

$p_{u}^{\text {macro }}$ : The transmit power of Macro

$p_{\text {ref }}^{\text {macro }}$ : The broadcast signals power from Macro over ABS subframes

$p_{u}^{\text {pico }}$ : The transmit power of Pico.

\section{Disclosure}

Ling Gao is now a part-time professor at Northwest University.

\section{Conflicts of Interest}

The authors declare that they have no conflicts of interest.

\section{Acknowledgments}

This work was supported in part by the National Natural Science Foundation of China (Grants nos. 61701400, 61501372, 61572401, 61373176, and 61672426), by the Natural Science Special Foundation of Education Department in Shaanxi (Grant no. 2017JQ6052), by the Postdoctoral Foundation of China (Grants nos. 2017M613188 and 2017M613186), and by Natural Science Special Foundation of Education Department in Shaanxi (Research on Joint Uplink and Downlink of Mobile Application "Performance-Power" Optimization).

\section{References}

[1] J. G. Andrews, H. Claussen, M. Dohler, S. Rangan, and M. C. Reed, "Femtocells: past, present, and future," IEEE Journal on Selected Areas in Communications, vol. 30, no. 3, pp. 497-508, 2012.

[2] Q. Ye, B. Rong, Y. Chen, M. Al-Shalash, C. Caramanis, and J. G. Andrews, "User association for load balancing in heterogeneous cellular networks," IEEE Transactions on Wireless Communications, vol. 12, no. 6, pp. 2706-2716, 2013.

[3] R. L. G. Cavalcante, S. Stańczak, M. Schubert, A. Eisenbläetter, and Ü. Tuerke, "Toward energy-efficient 5G wireless communications technologies: tools for decoupling the scaling of networks from the growth of operating power," IEEE Signal Processing Magazine, vol. 31, no. 6, pp. 24-34, 2014.

[4] S. Deb, P. Monogioudis, J. Miernik, and J. P. Seymour, "Algorithms for enhanced inter-cell interference coordination (eICIC) in LTE HetNets," IEEE/ACM Transactions on Networking, vol. 22, no. 1, pp. 137-150, 2014.

[5] M. Cierny, H. Wang, R. Wichman, Z. Ding, and C. Wijting, "On number of almost blank subframes in heterogeneous cellular networks," IEEE Transactions on Wireless Communications, vol. 12, no. 10, pp. 5061-5073, 2013.

[6] S. Vasudevan, R. N. Pupala, and K. Sivanesan, "Dynamic eICIC—a proactive strategy for improving spectral efficiencies of heterogeneous LTE cellular networks by leveraging user mobility and traffic dynamics," IEEE Transactions on Wireless Communications, vol. 12, no. 10, pp. 4956-4969, 2013.

[7] H. Zhou, Y. Ji, X. Wang, and S. Yamada, "Joint spectrum sharing and $\mathrm{ABS}$ adaptation for network virtualization in heterogeneous cellular networks," in Proceedings of the 58th IEEE Global Communications Conference, (GLOBECOM '15), IEEE, San Diego, CA, USA, December 2015.

[8] M. Wang, H. Xia, and C. Feng, "Joint eICIC and dynamic point blanking for energy-efficiency in heterogeneous network," in Proceedings of the International Conference on Wireless Communications and Signal Processing, (WCSP '15), pp. 1-6, IEEE, Nanjing, China, October 2015.

[9] Q. Kuang and W. Utschick, "Energy management in heterogeneous networks with cell activation, user association, and interference coordination," IEEE Transactions on Wireless Communications, vol. 15, no. 6, pp. 3868-3879, 2016.

[10] C. Yang, J. Li, Q. Ni, A. Anpalagan, and M. Guizani, "Interference-aware energy efficiency maximization in 5G ultra-dense networks," IEEE Transactions on Communications, vol. 65, no. 2, pp. 728-739, 2017.

[11] K. Son, H. Kim, Y. Yi, and B. Krishnamachari, "Base station operation and user association mechanisms for energy-delay tradeoffs in green cellular networks," IEEE Journal on Selected Areas in Communications, vol. 29, no. 8, pp. 1525-1536, 2011.

[12] S. Kim, S. Choi, and B. G. Lee, "A joint algorithm for base station operation and user association in heterogeneous networks," IEEE Communications Letters, vol. 17, no. 8, pp. 1552-1555, 2013.

[13] L. Su, C. Yang, Z. Xu, and A. F. Molisch, "Energy-efficient downlink transmission with base station closing in small cell networks," in Proceedings of the 38th IEEE International Conference on Acoustics, Speech, and Signal Processing (ICASSP '13), pp. 4784-4788, May 2013.

[14] T. Han and N. Ansari, "A traffic load balancing framework for software-defined radio access networks powered by hybrid energy sources," IEEE/ACM Transactions on Networking, vol. 24, no. 2, pp. 1038-1051, 2016.

[15] T. Han and N. Ansari, "Green-energy aware and latency aware user associations in heterogeneous cellular networks," in Proceedings of the IEEE Global Communications Conference (GLOBECOM '13), pp. 4946-4951, Atlanta, GA, USA, December 2013.

[16] B. Du, C. Pan, W. Zhang, and M. Chen, "Distributed energyefficient power optimization for CoMP systems with max-min fairness," IEEE Communications Letters, vol. 18, no. 6, pp. 9991002, 2014.

[17] X. Chen, H. Xia, Z. Zeng, S. Wu, W. Zuo, and Y. Lu, "Energyefficient heterogeneous networks for green communications by inter-layer interference coordination," in Proceedings of the 
2014 International Symposium on Wireless Personal Multimedia Communications, (WPMC '14), pp. 70-74, IEEE, Sydney, NSW, Australia, September 2014.

[18] B. Zhuang, D. Guo, and M. L. Honig, "Energy-efficient cell activation, user association, and spectrum allocation in heterogeneous networks," IEEE Journal on Selected Areas in Communications, vol. 34, no. 4, pp. 823-831, 2016.

[19] Q. Ye, M. Al-Shalashy, C. Caramanis, and J. G. Andrews, "On/off macrocells and load balancing in heterogeneous cellular networks," in Proceedings of the IEEE Global Communications Conference (GLOBECOM '13), pp. 3814-3819, IEEE, Atlanta, Ga, USA, December 2013.

[20] Y. Jia, M. Zhao, and W. Zhou, "Joint user association and eICIC for max-min fairness in hetnets," IEEE Communications Letters, vol. 20, no. 3, pp. 546-549, 2016.

[21] H. Zhou, Y. Ji, X. Wang, and S. Yamada, "EICIC configuration algorithm with service scalability in heterogeneous cellular networks," IEEE/ACM Transactions on Networking, pp. 1-6, 2016.

[22] J. Zheng, J. Li, N. Wang, and X. Yang, "Joint load balancing of downlink and uplink for eICIC in heterogeneous network," IEEE Transactions on Vehicular Technology, vol. 66, no. 7, pp. 6388-6398, 2017.

[23] R. J. Vanderbei, Linear Programming: Foundations and Extensions, Springer, 3rd edition, 2008.

[24] J.-P. Crouzeix and J. A. Ferland, "Algorithms for generalized fractional programming," Mathematical Programming, vol. 52, no. 2, pp. 191-207, 1991.

[25] J.-P. Crouzeix, J. A. Ferland, and S. Schaible, "An algorithm for generalized fractional programs," Journal of Optimization Theory and Applications, vol. 47, no. 1, pp. 35-49, 1985.

[26] S. Boyd and L. Vandenberghe, Convex Optimization, Cambridge University Press, 2004.

[27] L. Liberti and N. Maculan, Global Optimization, vol. 84, Kluwer Academic Publishers, Boston, Mass, USA, 2006. 


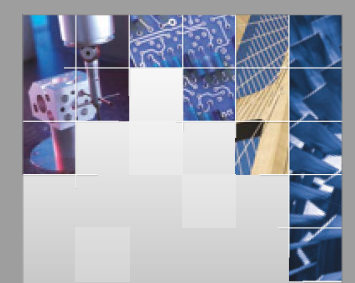

\section{Enfincering}
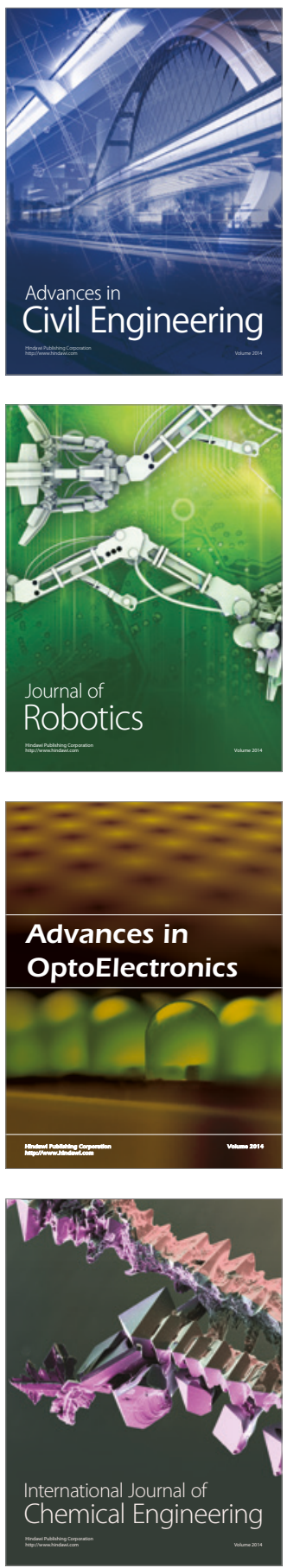

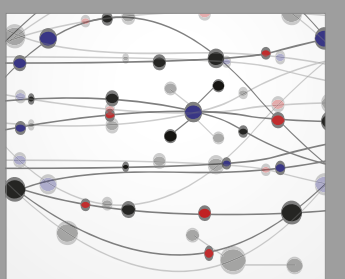

The Scientific World Journal

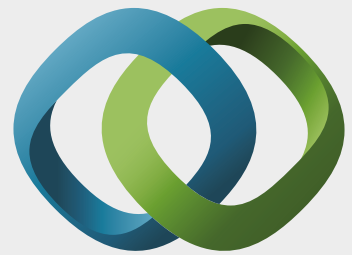

\section{Hindawi}

Submit your manuscripts at

https://www.hindawi.com
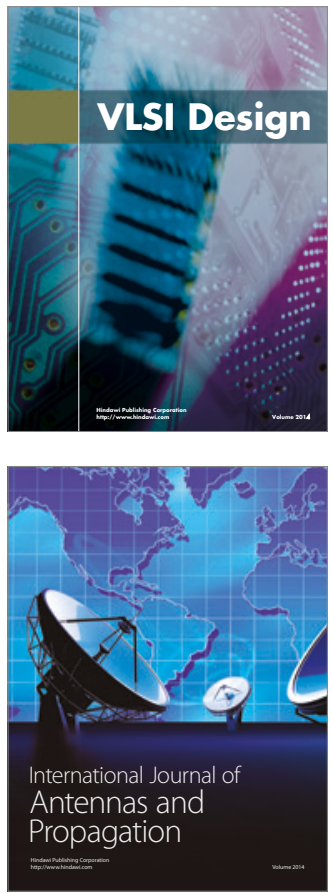

\section{Rotating}

Machinery
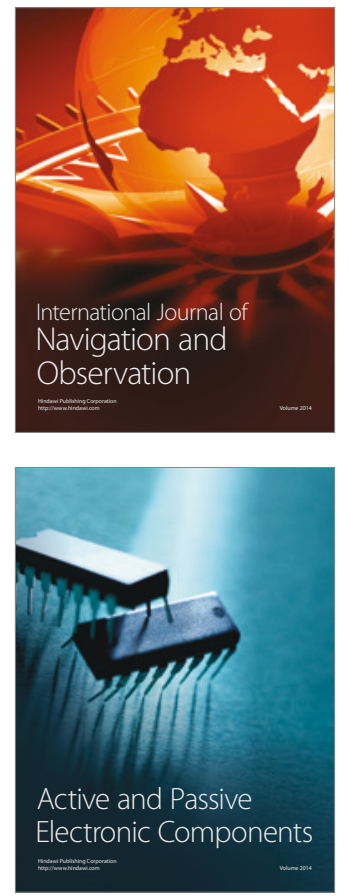
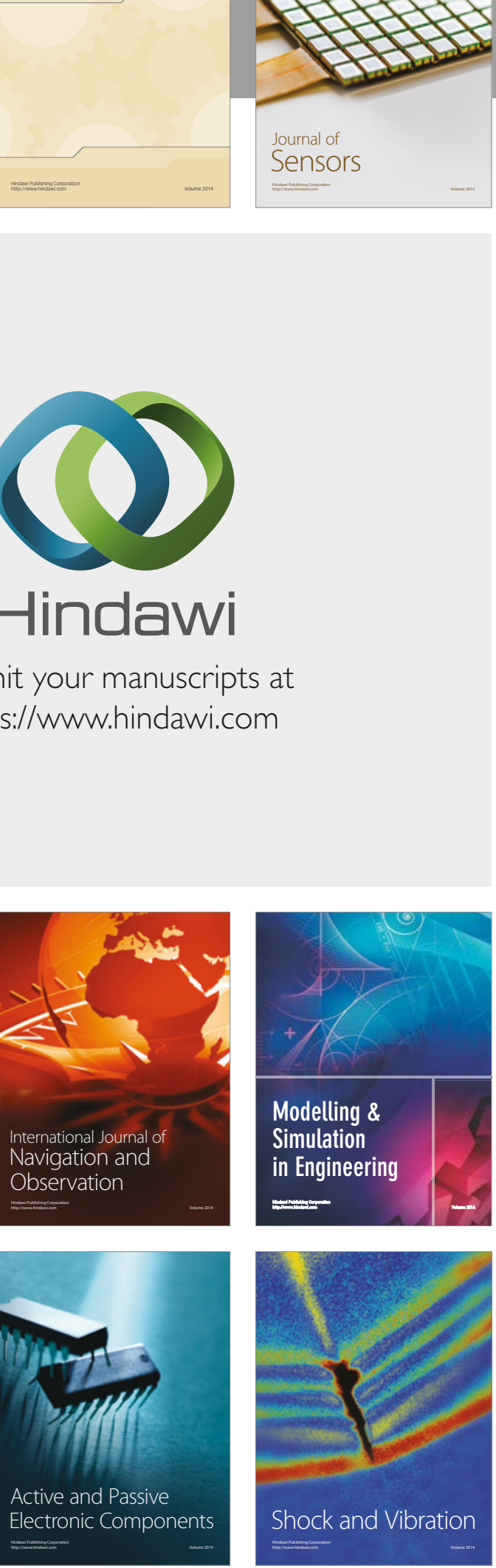
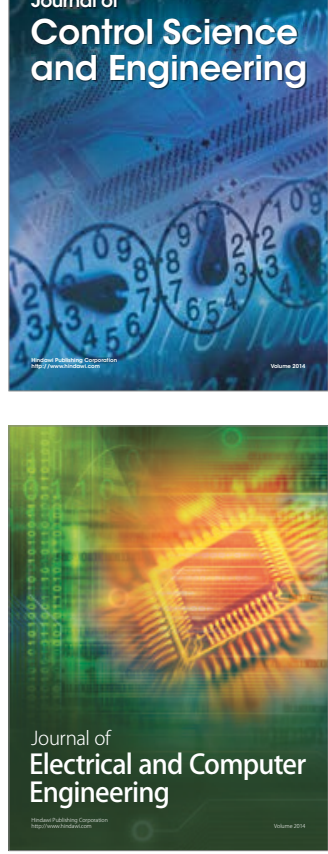

Distributed

Journal of

Control Science

and Engineering
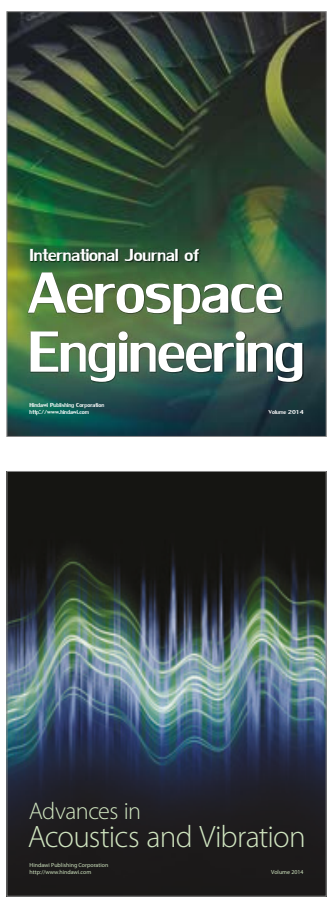

Sensor Networks 